\title{
Analytical Investigation of Surface Wave Characteristics of Piezoelectromagnetics of Class 6 mm
}

\author{
Aleksey A. Zakharenko \\ International Institute of Zakharenko Waves (IIZWs), Krasnoyarsk-37, Krasnoyarsk 17701, Russia \\ Correspondence should be addressed to Aleksey A. Zakharenko, aazaaz@inbox.ru
}

Received 13 March 2011; Accepted 22 May 2011

Academic Editors: Y.-D. Kwon and W. Yeih

Copyright (C) 2011 Aleksey A. Zakharenko. This is an open access article distributed under the Creative Commons Attribution License, which permits unrestricted use, distribution, and reproduction in any medium, provided the original work is properly cited.

This short work copes with theoretical investigations of some surface wave characteristics for transversely isotropic piezoelectromagnetic composites of class $6 \mathrm{~mm}$. In the composite materials, the surface Bleustein-Gulyaev-Melkumyan wave and some new shear-horizontal surface acoustic waves (SH-SAWs) recently discovered by the author can propagate. The phase velocities $V_{\mathrm{ph}}$ of the $\mathrm{SH}-\mathrm{SAWs}$ can have complicated dependencies on the coefficient of the magnetoelectromechanical coupling $K_{\mathrm{em}}^{2}(\mathrm{CMEMC})$ which depends on the electromagnetic constant $\alpha$ of the composites. Therefore, the analytical finding of the first and second partial derivatives of the $V_{\mathrm{ph}}(\alpha)$ represents the main purpose of this study. It is thought that the results of this short letter can help for theoreticians and experimentalists working in the research arena of opto-acoustoelectronics to completely understand some problems of surface wave propagation in piezoelectromagnetics.

\section{Introduction}

Two-phase composite materials, which possess both the piezoelectric and piezomagnetic phases, are very promising composites with the magnetoelectric effect. They are very interesting for various applications in space and aircraft technologies. Several books concerning composite materials are cited in [1-3]. The geometry of a two-phase composite material can be denoted by the following connectivities: 0-0, 0-1, 0-2, 0-3, 1-1, 1-2, 1-3, 2-2, 2-3, and 3-3, where $0,1,2$, and 3 are the dimensions of piezoelectric-piezomagnetic phases. Some latterly published papers concerning the magnetoelectric effect in different composite materials can be found in [4-9]. For example, the composite structures [10] called (2-2) composites represent laminated composite materials in which alternate layers of two different materials are bonded together to form a stratified continuum. Also, [10-13] cope with some laminate composites in which the popular Terfenol-D material is used as the piezomagnetic phase. For 
the study of the magnetoelectric effect, much work was described in the review paper [14] by Fiebig.

It is thought that some of the main characteristics of piezoelectric, piezomagnetic, and composite materials are the speeds of the shear-horizontal surface acoustic waves (SHSAWs). In 1998, Gulyaev [15] has written a review of SH-SAWs in solids. However, in the beginning of this millennium, Melkumyan [16] has discovered twelve new SH-SAWs in piezoelectromagnetic composite materials. In 2010, the author of this paper has additionally discovered seven new SH-SAWs in the piezoelectromagnetic composites of class $6 \mathrm{~mm}$, see the book [17]. One of the new surface Melkumyan waves [16] written in the following section was called the surface Bleustein-Gulyaev-Melkumyan wave [17]. Note that the classical SHSAWs in purely piezoelectric materials and purely piezomagnetic materials are called the surface Bleustein-Gulyaev waves simultaneously discovered by Bleustein [18] and Gulyaev [19] to the end of the last millennium. The new SH-SAWs discovered in [17] by the author of this theoretical work depend on the speed of light in a vacuum and can represent an interest for acoustooptics and photonics researchers. Also, some peculiarities of the new SH-SAW propagation will be briefly discussed in the following section. This peculiarity allows one to assume a restriction for the electromagnetic constant $\alpha$ of the complex piezoelectromagnetic composite materials.

It is also noted that SH-SAWs can easily be produced by electromagnetic acoustic transducers (EMATs), a nontrivial task for common piezoelectric transducers [20]. The EMATs can offer advantages over traditional piezoelectric transducers. Comprehensive monographs [21,22] on the EMATs collect the research activities on this topic. Therefore, it is thought that this short theoretical work can be also useful as a small step towards new applications of the EMATs technologies. Indeed, it is believed that some characteristics of the SH-SAWs in piezoelectromagnetic composite materials can be revealed by the utilization of the electromagnetic acoustic transducers. Therefore, the following section describes the analytical finding of the first and second partial derivatives of the phase velocity $V_{\mathrm{ph}}$ with respect to the electromagnetic constant $\alpha$.

\section{Theoretical Investigations}

According to the recent work by Melkumyan [16] concerning wave propagation in piezoelectromagnetic materials of class $6 \mathrm{~mm}$, the velocity $V_{\mathrm{BGM}}$ for the shear-horizontal surface Bleustein-Gulyaev-Melkumyan wave can be written in the explicit form [17] as follows:

$$
V_{\mathrm{BGM}}=V_{\mathrm{tem}}\left[1-\left(\frac{K_{\mathrm{em}}^{2}}{1+K_{\mathrm{em}}^{2}}\right)^{2}\right]^{1 / 2} .
$$

In (2.1), the velocity $V_{\text {tem }}$ of the piezo-magnetoelectromechanical shear-horizontal bulk acoustic wave (SH-BAW) and the coefficient of the magnetoelectromechanical coupling $K_{\mathrm{em}}^{2}$ (CMEMC) are defined as follows:

$$
\begin{gathered}
V_{\mathrm{tem}}=V_{t 4}\left(1+K_{\mathrm{em}}^{2}\right)^{1 / 2}, \\
K_{\mathrm{em}}^{2}=\frac{\mu e^{2}+\varepsilon h^{2}-2 \alpha e h}{C\left(\varepsilon \mu-\alpha^{2}\right)} .
\end{gathered}
$$


In (2.2), the velocity $V_{t 4}$ of the purely mechanical SH-BAW is determined as follows:

$$
V_{t 4}=\sqrt{\frac{C}{\rho}},
$$

where $\rho$ is the mass density. In (2.3) and (2.4), there are the following material constants: the elastic stiffness constant $C$, piezoelectric constant $e$, piezomagnetic coefficient $h$, dielectric permittivity coefficient $\varepsilon$, magnetic permeability coefficient $\mu$, and electromagnetic constant $\alpha$. The material constants are described in the well-known handbook [23] on electromagnetic materials.

Formula (2.1) for the surface Bleustein-Gulyaev-Melkumyan (BGM) wave corresponds to the first case of the electrical and magnetic boundary conditions at the interface between the composite surface and a vacuum. This case is for the electrically closed surface (electrical potential $\varphi=0$ ) and the magnetically open surface (magnetic potential $\psi=0$ ) using the mechanical boundary condition of the mechanically free interface. The realization of different boundary conditions is described in an excellent theoretical work [24]. In addition to the first case, it is also possible to treat the second case of electrical and magnetic boundary conditions for the mechanical boundary condition. This second case represents the continuity of both the normal components of $D_{3}$ and $B_{3}$ at the interface, where $D_{3}$ and $B_{3}$ are the components of the electrical displacement and the magnetic flux, respectively. This leads to the following velocities for the SH-SAWs discovered by the author in the recent theoretical work [17]:

$$
\begin{gathered}
V_{\text {new } 1}=V_{\text {tem }}\left[1-\left(\frac{K_{\mathrm{em}}^{2}-K_{e}^{2}+\alpha^{2} C_{L}^{2}\left(\varepsilon_{0} / \varepsilon\right)\left(K_{\mathrm{em}}^{2}-e h / \alpha C\right)}{\left(1+K_{\mathrm{em}}^{2}\right)\left(1+\mu / \mu_{0}\right)}\right)^{2}\right]^{1 / 2}, \\
V_{\text {new } 2}=V_{\text {tem }}\left[1-\left(\frac{K_{\mathrm{em}}^{2}-K_{m}^{2}+\alpha^{2} C_{L}^{2}\left(\mu_{0} / \mu\right)\left(K_{\mathrm{em}}^{2}-e h / \alpha C\right)}{\left(1+K_{\mathrm{em}}^{2}\right)\left(1+\varepsilon / \varepsilon_{0}\right)}\right)^{2}\right]^{1 / 2} .
\end{gathered}
$$

In expressions (2.5) and (2.6) there is already dependence on the vacuum characteristics such as the dielectric permittivity constant $\varepsilon_{0}=10^{-7} /\left(4 \pi C_{L}^{2}\right)=8.854187817 \times 10^{-12}[\mathrm{~F} / \mathrm{m}]$ and the magnetic permeability constant $\mu_{0}=4 \pi \times 10^{-7}[\mathrm{H} / \mathrm{m}]=12.5663706144 \times 10^{-7}[\mathrm{H} / \mathrm{m}]$, where $C_{L}=2.99782458 \times 10^{8}[\mathrm{~m} / \mathrm{s}]$ is the speed of light in a vacuum:

$$
C_{L}^{2}=\frac{1}{\varepsilon_{0} \mu_{0}}
$$

Also, expression (2.5) depends on the well-known coefficient of the electromechanical coupling $K_{e}^{2}(\mathrm{CEMC})$ for a purely piezoelectric material (see below), and expression (2.6) 
depends on the well-known coefficient of the magnetomechanical coupling $K_{m}^{2}$ (CMMC) for a purely piezomagnetic material:

$$
\begin{aligned}
K_{e}^{2} & =\frac{e^{2}}{\varepsilon C^{\prime}}, \\
K_{m}^{2} & =\frac{h^{2}}{\mu C} .
\end{aligned}
$$

Therefore, it is possible to obtain the first and second derivatives of the velocities $V_{\mathrm{BGM}}$, $V_{\text {new1 }}$, and $V_{\text {new2 }}$ with respect to the electromagnetic constant $\alpha$ as the results of the theoretical investigations for this short report. Note that these investigations were not carried out in the recent book [17] due to some mathematical complexities. Therefore, this report continues the theoretical investigations of the book [17]. These investigations are useful because it is possible that the functions $V_{\mathrm{BGM}}(\alpha>0), V_{\text {new } 1}(\alpha>0)$, and $V_{\text {new2 }}(\alpha>0)$ can have some peculiarities, namely, the SH-SAWs cannot exist for some large values of $\alpha^{2} \rightarrow \varepsilon \mu$ when $K_{\mathrm{em}}^{2} \rightarrow \infty$; see formula $(2.3)$. Note that papers $[25,26]$ studied some composites with the electromagnetic constant $\alpha<0$, for which these peculiarities do not exist. Therefore, it allows one to suppose that the right sign for the electromagnetic constant $\alpha$ is negative.

The first partial derivatives of the velocities $V_{\mathrm{BGM}}, V_{\text {new1 }}$, and $V_{\text {new2 }}$ with respect to the constant $\alpha$ can be written in the following forms:

$$
\begin{gathered}
\frac{\partial V_{\mathrm{BGM}}}{\partial \alpha}=\frac{V_{\mathrm{BGM}}}{V_{\mathrm{tem}}} \frac{\partial V_{\mathrm{tem}}}{\partial \alpha}-\frac{V_{\mathrm{tem}}^{2}}{V_{\mathrm{BGM}}} \frac{K_{\mathrm{em}}^{2}}{\left(1+K_{\mathrm{em}}^{2}\right)^{3}} \frac{\partial K_{\mathrm{em}}^{2}}{\partial \alpha}, \\
\frac{\partial V_{\text {new } 1}}{\partial \alpha}=\frac{V_{\text {new } 1}}{V_{\text {tem }}} \frac{\partial V_{\text {tem }}}{\partial \alpha}-\frac{b_{1} V_{\text {tem }}^{2}}{V_{\text {new } 1}} \frac{\partial b_{1}}{\partial \alpha}, \\
\frac{\partial V_{\text {new } 2}}{\partial \alpha}=\frac{V_{\text {new } 2}}{V_{\text {tem }}} \frac{\partial V_{\text {tem }}}{\partial \alpha}-\frac{b_{2} V_{\text {tem }}^{2}}{V_{\text {new } 2}} \frac{\partial b_{2}}{\partial \alpha}
\end{gathered}
$$

where

$$
\frac{\partial V_{\mathrm{tem}}}{\partial \alpha}=\frac{V_{t 4}^{2}}{2 V_{\mathrm{tem}}} \frac{\partial K_{\mathrm{em}}^{2}}{\partial \alpha}
$$

In (2.9)-(2.12), the first partial derivative of the CMEMC $K_{\mathrm{em}}^{2}$ with respect to the electromagnetic constant $\alpha$ is defined by

$$
\frac{\partial K_{\mathrm{em}}^{2}}{\partial \alpha}=\frac{2\left(\alpha K_{\mathrm{em}}^{2}-e h / C\right)}{\varepsilon \mu-\alpha^{2}} .
$$



follows:

Using (2.5) and (2.6), the functions $b_{1}$ and $b_{2}$ in (2.10) and (2.11) are determined as

$$
\begin{gathered}
b_{1}=\frac{K_{\mathrm{em}}^{2}-K_{e}^{2}+\alpha^{2} C_{L}^{2}\left(\varepsilon_{0} / \varepsilon\right)\left(K_{\mathrm{em}}^{2}-e h / \alpha C\right)}{\left(1+K_{\mathrm{em}}^{2}\right)\left(1+\mu / \mu_{0}\right)}, \\
b_{2}=\frac{K_{\mathrm{em}}^{2}-K_{m}^{2}+\alpha^{2} C_{L}^{2}\left(\mu_{0} / \mu\right)\left(K_{\mathrm{em}}^{2}-e h / \alpha C\right)}{\left(1+K_{\mathrm{em}}^{2}\right)\left(1+\varepsilon / \varepsilon_{0}\right)} .
\end{gathered}
$$

Therefore, the first partial derivatives of the $b_{1}$ and $b_{2}$ with respect to the constant $\alpha$ can be expressed in the following forms:

$$
\begin{aligned}
\frac{\partial b_{1}}{\partial \alpha}= & \frac{\left[1-b_{1}\left(1+\mu / \mu_{0}\right)\right]\left(\partial K_{\mathrm{em}}^{2} / \partial \alpha\right)+2 \alpha C_{L}^{2}\left(\varepsilon_{0} / \varepsilon\right)\left(K_{\mathrm{em}}^{2}-e h / \alpha C\right)}{\left(1+K_{\mathrm{em}}^{2}\right)\left(1+\mu / \mu_{0}\right)} \\
& +\frac{\alpha^{2} C_{L}^{2}\left(\varepsilon_{0} / \varepsilon\right)\left(\partial K_{\mathrm{em}}^{2} / \partial \alpha+e h / \alpha^{2} C\right)}{\left(1+K_{\mathrm{em}}^{2}\right)\left(1+\mu / \mu_{0}\right)} \\
\frac{\partial b_{2}}{\partial \alpha}= & \frac{\left[1-b_{2}\left(1+\varepsilon / \varepsilon_{0}\right)\right]\left(\partial K_{\mathrm{em}}^{2} / \partial \alpha\right)+2 \alpha C_{L}^{2}\left(\mu_{0} / \mu\right)\left(K_{\mathrm{em}}^{2}-e h / \alpha C\right)}{\left(1+K_{\mathrm{em}}^{2}\right)\left(1+\varepsilon / \varepsilon_{0}\right)} \\
& +\frac{\alpha^{2} C_{L}^{2}\left(\mu_{0} / \mu\right)\left(\left(\partial K_{\mathrm{em}}^{2} / \partial \alpha\right)+\left(e h / \alpha^{2} C\right)\right)}{\left(1+K_{\mathrm{em}}^{2}\right)\left(1+\varepsilon / \varepsilon_{0}\right)} .
\end{aligned}
$$

The second partial derivatives of the velocities $V_{\mathrm{BGM}}, V_{\text {new1 }}$, and $V_{\text {new2 }}$ with respect to the electromagnetic constant $\alpha$ read

$$
\begin{aligned}
\frac{\partial^{2} V_{\mathrm{BGM}}}{\partial \alpha^{2}}= & \frac{V_{\mathrm{BGM}}}{V_{\mathrm{tem}}} \frac{\partial^{2} V_{\mathrm{tem}}}{\partial \alpha^{2}}+\frac{1}{V_{\mathrm{tem}}} \frac{\partial V_{\mathrm{BGM}}}{\partial \alpha} \frac{\partial V_{\mathrm{tem}}}{\partial \alpha}-\frac{V_{\mathrm{BGM}}}{V_{\mathrm{tem}}^{2}}\left(\frac{\partial V_{\mathrm{tem}}}{\partial \alpha}\right)^{2}-\frac{V_{\mathrm{tem}}^{2}}{V_{\mathrm{BGM}}} \frac{K_{\mathrm{em}}^{2}}{\left(1+K_{\mathrm{em}}^{2}\right)^{3}} \frac{\partial^{2} K_{\mathrm{em}}^{2}}{\partial \alpha^{2}} \\
& -\left[2 \frac{V_{\mathrm{tem}}}{V_{\mathrm{BGM}}} \frac{\partial V_{\mathrm{tem}}}{\partial \alpha}-\left(\frac{V_{\mathrm{tem}}}{V_{\mathrm{BGM}}}\right)^{2} \frac{\partial V_{\mathrm{BGM}}}{\partial \alpha}\right] \frac{K_{\mathrm{em}}^{2}}{\left(1+K_{\mathrm{em}}^{2}\right)^{3}} \frac{\partial K_{\mathrm{em}}^{2}}{\partial \alpha} \\
& -\left(1-\frac{3 K_{\mathrm{em}}^{2}}{1+K_{\mathrm{em}}^{2}}\right) \frac{V_{\mathrm{tem}}^{2}}{V_{\mathrm{BGM}}} \frac{1}{\left(1+K_{\mathrm{em}}^{2}\right)^{3}}\left(\frac{\partial K_{\mathrm{em}}^{2}}{\partial \alpha}\right)^{2},
\end{aligned}
$$$$
\frac{\partial^{2} V_{\text {new } 1}}{\partial \alpha^{2}}=\frac{V_{\text {new } 1}}{V_{\text {tem }}} \frac{\partial^{2} V_{\text {tem }}}{\partial \alpha^{2}}-\frac{V_{\text {tem }}^{2}}{V_{\text {new } 1}}\left(\frac{\partial b_{1}}{\partial \alpha}\right)^{2}-b_{1} \frac{V_{\text {tem }}^{2}}{V_{\text {new } 1}} \frac{\partial^{2} b_{1}}{\partial \alpha^{2}}
$$$$
-3 b_{1} \frac{V_{\text {tem }}}{V_{\text {new } 1}} \frac{\partial V_{\text {tem }}}{\partial \alpha} \frac{\partial b_{1}}{\partial \alpha}+b_{1}\left(\frac{V_{\text {tem }}}{V_{\text {new } 1}}\right)^{2} \frac{\partial V_{\text {new } 1}}{\partial \alpha} \frac{\partial b_{1}}{\partial \alpha}
$$ 


$$
\begin{aligned}
\frac{\partial^{2} V_{\text {new } 2}}{\partial \alpha^{2}}= & \frac{V_{\text {new } 2}}{V_{\text {tem }}} \frac{\partial^{2} V_{\text {tem }}}{\partial \alpha^{2}}-\frac{V_{\text {tem }}^{2}}{V_{\text {new2 }}}\left(\frac{\partial b_{2}}{\partial \alpha}\right)^{2}-b_{2} \frac{V_{\text {tem }}^{2}}{V_{\text {new } 2}} \frac{\partial^{2} b_{2}}{\partial \alpha^{2}} \\
& -3 b_{2} \frac{V_{\text {tem }}}{V_{\text {new2 }}} \frac{\partial V_{\text {tem }}}{\partial \alpha} \frac{\partial b_{2}}{\partial \alpha}+b_{2}\left(\frac{V_{\text {tem }}}{V_{\text {new2 }}}\right)^{2} \frac{\partial V_{\text {new } 2}}{\partial \alpha} \frac{\partial b_{2}}{\partial \alpha},
\end{aligned}
$$

where

$$
\frac{\partial^{2} V_{\mathrm{tem}}}{\partial \alpha^{2}}=\frac{V_{t 4}^{2}}{2 V_{\mathrm{tem}}} \frac{\partial^{2} K_{\mathrm{em}}^{2}}{\partial \alpha^{2}}-\frac{V_{t 4}^{2}}{2 V_{\mathrm{tem}}^{2}} \frac{\partial V_{\mathrm{tem}}}{\partial \alpha} \frac{\partial K_{\mathrm{em}}^{2}}{\partial \alpha} .
$$

In (2.16) and (2.19), the second partial derivative of the $K_{\mathrm{em}}^{2}$ with respect to the constant $\alpha$ is defined as follows:

$$
\frac{\partial^{2} K_{\mathrm{em}}^{2}}{\partial \alpha^{2}}=\frac{2 K_{\mathrm{em}}^{2}+4 \alpha\left(\partial K_{\mathrm{em}}^{2} / \partial \alpha\right)}{\varepsilon \mu-\alpha^{2}} .
$$

In (2.17) and (2.18), the second partial derivatives of the $b_{1}$ and $b_{2}$ with respect to the $\alpha$ are

$$
\begin{aligned}
\frac{\partial^{2} b_{1}}{\partial \alpha^{2}} & =\frac{B_{1}}{\left(1+K_{\mathrm{em}}^{2}\right)\left(1+\mu / \mu_{0}\right)}, \\
\frac{\partial^{2} b_{2}}{\partial \alpha^{2}} & =\frac{B_{2}}{\left(1+K_{\mathrm{em}}^{2}\right)\left(1+\varepsilon / \varepsilon_{0}\right)},
\end{aligned}
$$

where

$$
\begin{aligned}
B_{1}= & {\left[1-b_{1}\left(1+\frac{\mu}{\mu_{0}}\right)\right] \frac{\partial^{2} K_{\mathrm{em}}^{2}}{\partial \alpha^{2}}-2\left(1+\frac{\mu}{\mu_{0}}\right) \frac{\partial b_{1}}{\partial \alpha} \frac{\partial K_{\mathrm{em}}^{2}}{\partial \alpha}+2 C_{L}^{2} \frac{\varepsilon_{0}}{\varepsilon}\left(K_{\mathrm{em}}^{2}-\frac{e h}{\alpha C}\right) } \\
& +4 \alpha C_{L}^{2} \frac{\varepsilon_{0}}{\varepsilon}\left(\frac{\partial K_{\mathrm{em}}^{2}}{\partial \alpha}+\frac{e h}{\alpha^{2} C}\right)+\alpha^{2} C_{L}^{2} \frac{\varepsilon_{0}}{\varepsilon}\left(\frac{\partial^{2} K_{\mathrm{em}}^{2}}{\partial \alpha^{2}}-\frac{2 e h}{\alpha^{3} C}\right), \\
B_{2}= & {\left[1-b_{2}\left(1+\frac{\varepsilon}{\varepsilon_{0}}\right)\right] \frac{\partial^{2} K_{\mathrm{em}}^{2}}{\partial \alpha^{2}}-2\left(1+\frac{\varepsilon}{\varepsilon_{0}}\right) \frac{\partial b_{2}}{\partial \alpha} \frac{\partial K_{\mathrm{em}}^{2}}{\partial \alpha}+2 C_{L}^{2} \frac{\mu_{0}}{\mu}\left(K_{\mathrm{em}}^{2}-\frac{e h}{\alpha C}\right) } \\
& +4 \alpha C_{L}^{2} \frac{\mu_{0}}{\mu}\left(\frac{\partial K_{\mathrm{em}}^{2}}{\partial \alpha}+\frac{e h}{\alpha^{2} C}\right)+\alpha^{2} C_{L}^{2} \frac{\mu_{0}}{\mu}\left(\frac{\partial^{2} K_{\mathrm{em}}^{2}}{\partial \alpha^{2}}-\frac{2 e h}{\alpha^{3} C}\right) .
\end{aligned}
$$

It is obvious that the first partial derivatives of the velocities $V_{\mathrm{BGM}}, V_{\text {new1 }}$, and $V_{\text {new2 }}$ with respect to the electromagnetic constant $\alpha$ have dimension of $(\mathrm{m} / \mathrm{s})^{2}$ and can represent some squares in the corresponding two-dimensional (2D) spaces of velocities. Analogically, the second partial derivatives of the velocities with respect to the constant $\alpha$ can represent some volumes with dimensions of $(\mathrm{m} / \mathrm{s})^{3}$ in the corresponding 3D spaces of velocities. Indeed, it is also possible to graphically investigate the complicated first and second partial derivatives of the velocities obtained in formulae (2.9)-(2.11) and from (2.16) to (2.18). However, this does not represent the purpose of this short report. 


\section{Conclusion}

This short theoretical report further developed the study of the recently published book [17]. In this work, on some wave properties of composite materials, the propagation peculiarities of new shear-horizontal surface acoustic waves (SH-SAWs) recently discovered in book [17] were theoretically studied and briefly discussed. Therefore, the analytical finding of the first and second partial derivatives of the phase velocity with respect to the electromagnetic constant $\alpha$ represented the main purpose of this study. This theoretical work can be useful for theoreticians and experimentalists working in the arena of acoustooptics, photonics, and opto-acoustoelectronics. Also, the theoretical study of this short paper can be useful for investigations of cubic piezoelectromagnetics like the researches carried out for cubic piezoelectrics [27] and cubic piezomagnetics [28].

\section{Acknowledgment}

The author would like to thank the referees for useful notes.

\section{References}

[1] A. K. Kaw, Mechanics of Composite Materials, CRC Press, New York, NY, USA, 2005.

[2] L. Hollaway, Handbook of Polymer Composites for Engineers, Woodhead Publishing, Cambridge, UK, 1994.

[3] F. L. Matthews and R. D. Rawlings, Composite Materials: Engineering and Science, CRC Press, Boca Raton, Fla, USA, 1999.

[4] M. Zeng, S. W. Or, and H. L. W. Wa Chan, "Magnetic field-induced strain and magnetoelectric effects in sandwich composite of ferromagnetic shape memory Ni-Mn-Ga crystal and piezoelectric PVDF polymer," IEEE Transactions on Ultrasonics, Ferroelectrics, and Frequency Control, vol. 57, no. 10, article 5587392, pp. 2147-2153, 2010.

[5] M.-S Guo and S. Dong, "A resonance-bending mode magnetoelectric-coupling equivalent circuit," IEEE Transactions on Ultrasonics, Ferroelectrics, and Frequency Control, vol. 56, no. 11, Article ID 5306739, pp. 2578-2586, 2009.

[6] M.-S. Guo and Sh.-X. Dong, "Annular bilayer magnetoelectric composites: theoretical analysis," IEEE Transactions on Ultrasonics, Ferroelectrics, and Frequency Control, vol. 57, no. 2, pp. 480-489, 2010.

[7] Ü. Özgür, Y. Alivov, and H. Morkoç, "Microwave ferrites, part 2: passive components and electrical tuning," Journal of Materials Science: Materials in Electronics, vol. 20, no. 10, pp. 911-952, 2009.

[8] M. Zeng, S. W. Or, and H. L. W. Chan, "DC- and ac-magnetic field induced strain effects in ferromagnetic shape memory composites of Ni-Mn-Ga single crystal and polyurethane polymer," Journal of Applied Physics, vol. 107, article 09A942, 2010.

[9] J. X. Zhang, J. Y. Dai, L. C. So et al., "The effect of magnetic nanoparticles on the morphology, ferroelectric, and magnetoelectric behaviors of CFO/P(VDF-TrFE) 0-3 nanocomposites," Journal of Applied Physics, vol. 105, no. 5 article 054102, 2009.

[10] M. Avellaneda and G. Harshe, "Magnetoelectric effect in piezoelectric/magnetostrictive multilayer (2-2) composites," Journal of Intelligent Material Systems and Structures, vol. 5, no. 4, pp. 501-513, 1994.

[11] S.-X Dong, J. F. Li, and D. Viehland, "Longitudinal and transverse magnetoelectric voltage coefficients of magnetostrictive/piezoelectric laminate composite: experiments," IEEE Transactions on Ultrasonics, Ferroelectrics, and Frequency Control, vol. 51, no. 7, pp. 794-799, 2004.

[12] S. S. Guo, S. G. Lu, Z. Xu, X. Z. Zhao, and S. W. Or, "Enhanced magnetoelectric effect in Terfenol-D and flextensional cymbal laminates," Applied Physics Letters, vol. 88, no. 18, article 182906, 2006.

[13] Y. Wang, S. W. Or, H. L. W. Chan, X. Zhao, and H. Luo, "Enhanced magnetoelectric effect in longitudinal-transverse mode Terfenol- $\mathrm{D} / \mathrm{Pb}\left(\mathrm{Mg}_{1 / 3} \mathrm{Nb}_{2 / 3}\right) \mathrm{O}_{3}-\mathrm{PbTiO}_{3}$ laminate composites with optimal crystal cut," Journal of Applied Physics, vol. 103, no. 12, article 124511, 2008.

[14] M. Fiebig, "Revival of the magnetoelectric effect," Journal of Physics D, vol. 38, no. 8, pp. R123-R152, 2005. 
[15] Y. V. Gulyaev, "Review of shear surface acoustic waves in solids," IEEE Transactions on Ultrasonics, Ferroelectrics, and Frequency Control, vol. 45, no. 4, pp. 935-938, 1998.

[16] A. Melkumyan, "Twelve shear surface waves guided by clamped/free boundaries in magneto-electro-elastic materials," International Journal of Solids and Structures, vol. 44, no. 10, pp. 3594-3599, 2007.

[17] A. A. Zakharenko, Propagation of Seven new SH-SAWs in Piezoelectromagnetics of Class 6 mm, Laplambert Academic Publishing GmbH \& Co. KG, Saarbrücken, Germany, 2010.

[18] J. L. Bleustein, "A new surface wave in piezoelectric materials," Applied Physics Letters, vol. 13, no. 12, pp. 412-413, 1968.

[19] Y. V. Gulyaev, "Electroacoustic surface waves in solids," Soviet Physics Journal of Experimental and Theoretical Physics Letters, vol. 9, pp. 37-38, 1969.

[20] R. Ribichini, F. Cegla, P. B. Nagy, and P. Cawley, "Quantitative modeling of the transduction of electromagnetic acoustic transducers operating on ferromagnetic media," IEEE Transactions on Ultrasonics, Ferroelectrics, and Frequency Control, vol. 57, no. 12, Article ID 5610566, pp. 2808-2817, 2010.

[21] R. B. Thompson, "Physical principles of measurements with EMAT transducers," in Physical Acoustics, W. P. Mason and R. N. Thurston, Eds., vol. 19, pp. 157-200, Academic Press, New York, NY, USA, 1990.

[22] M. Hirao and H. Ogi, EMATs for Science and Industry: Non-contacting Ultrasonic Measurements, Kluwer Academic, Boston, Mass, USA, 2003.

[23] P. S. Neelakanta, Handbook of Electromagnetic Materials, CRC Press, New York, NY, USA, 1995.

[24] V. I. Al'shits, A. N. Darinskii, and J. Lothe, "On the existence of surface waves in half-infinite anisotropic elastic media with piezoelectric and piezomagnetic properties," Wave Motion, vol. 16, no. 3, pp. 265-283, 1992.

[25] J. Y. Li, "Magnetoelectroelastic multi-inclusion and inhomogeneity problems and their applications in composite materials," International Journal of Engineering Science, vol. 38, no. 18, pp. 1993-2011, 2000.

[26] X.-F. Li, "Dynamic analysis of a cracked magnetoelectroelastic medium under antiplane mechanical and inplane electric and magnetic impacts," International Journal of Solids and Structures, vol. 42, no. 11-12, pp. 3185-3205, 2005.

[27] A. A. Zakharenko, Dispersive SAWs in Layered Systems Consisting of Cubic Piezoelectrics, Laplambert Academic Publishing GmbH \& Co. KG, Saarbrücken, Germany, 2010.

[28] A. A. Zakharenko, "First evidence of surface SH-wave propagation in cubic piezomagnetics," Journal of Electromagnetic Analysis and Applications, vol. 2, no. 5, pp. 287-296, 2010. 


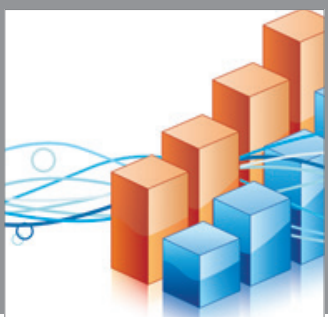

Advances in

Operations Research

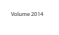

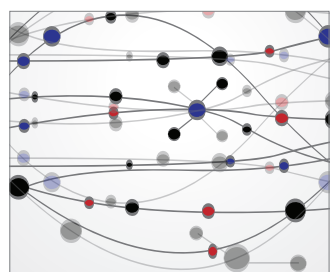

\section{The Scientific} World Journal
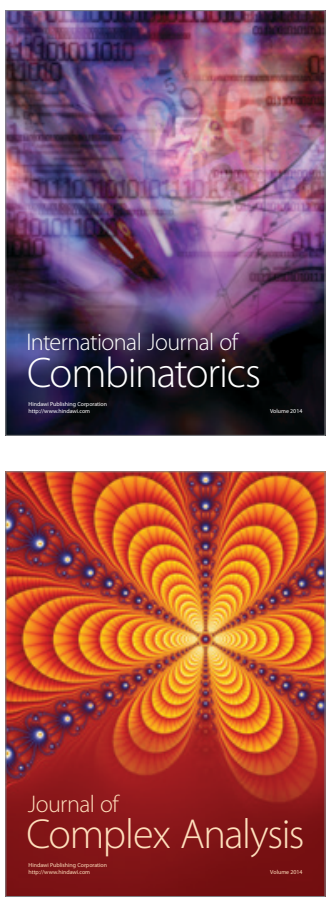

International Journal of

Mathematics and

Mathematical

Sciences
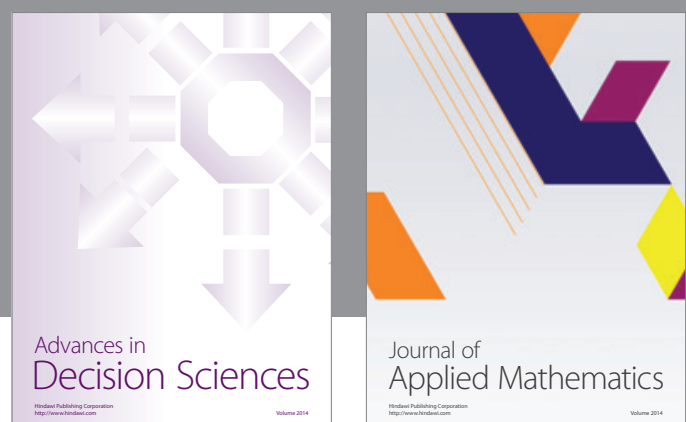

Journal of

Applied Mathematics
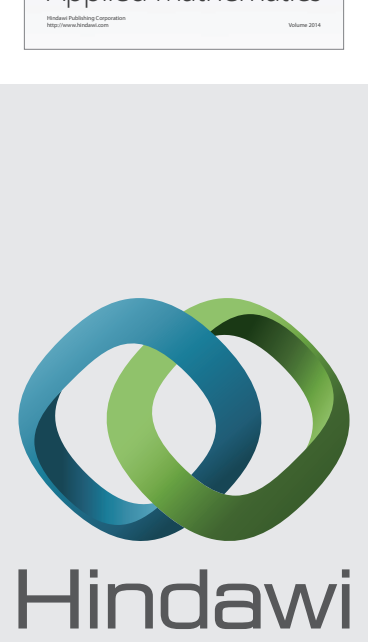

Submit your manuscripts at http://www.hindawi.com
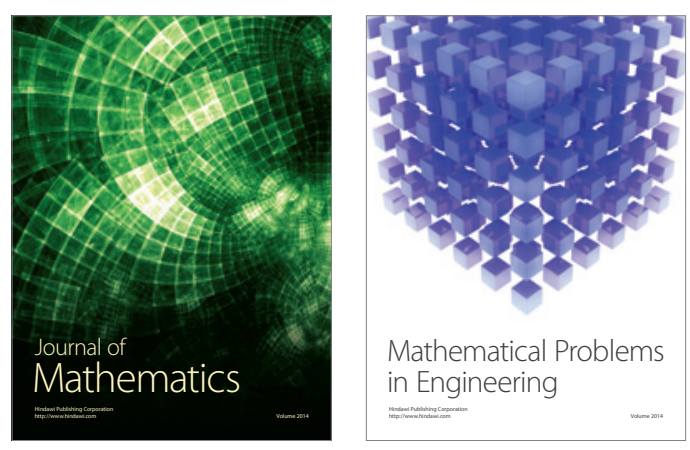

Mathematical Problems in Engineering
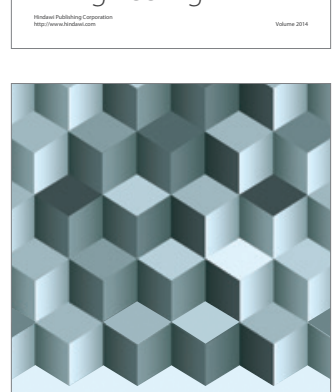

Journal of

Function Spaces
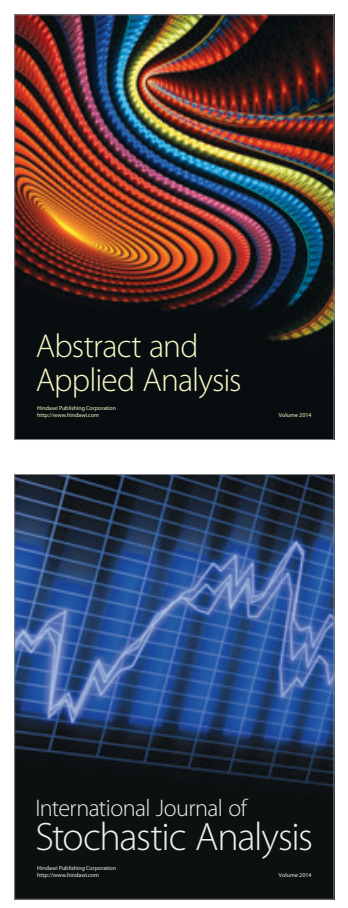

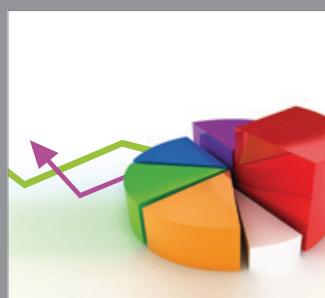

ournal of

Probability and Statistics

Promensencen
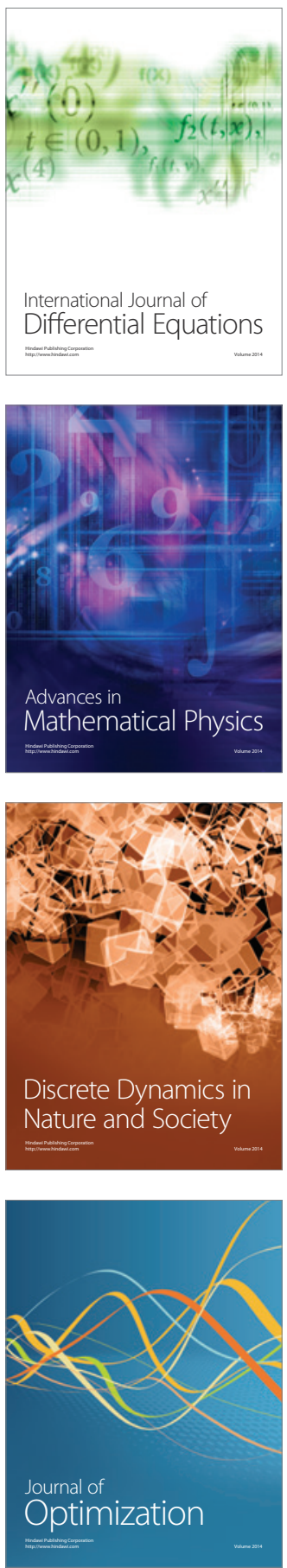